\title{
The energy-momentum tensor and D-term of Q-clouds
}

\author{
Michael Cantara ${ }^{\mathrm{a}}$, Manuel Mai ${ }^{\mathrm{b}}$, Peter Schweitzer ${ }^{\mathrm{a}}$ \\ ${ }^{a}$ Department of Physics, University of Connecticut, Storrs, CT 06269-3046, U.S.A. \\ ${ }^{b}$ Department of Physics Yale University, New Haven, CT 06511-8499, U.S.A.
}

\begin{abstract}
The $D$-term is, like mass and spin, a fundamental property related to the energymomentum tensor. Yet it is not known experimentally for any particle. In all theoretical studies so far the $D$-terms of various particles were found negative. Early works gave rise to the assumption the negative sign could be related to stability. The emerging question is whether it is possible to find a field-theoretical system with a positive $D$-term. To shed some light on this question we investigate $Q$-clouds, an extreme parametric limit in the $Q$-ball system. $Q$-clouds are classically unstable solutions which delocalize, spread out over all space forming an infinitely dilute gas of free quanta, and are even energetically unstable against tunneling to plane waves. In short, these extremely unstable field configurations provide an ideal candidate system for our purposes. By studying the energy-momentum tensor we show that at any stage of the $Q$-cloud limit one deals with perfectly well-defined and, when viewed in appropriately scaled coordinates, non-dissipating non-topological solitonic solutions. We investigate in detail their properties, and find new physical interpretations by observing that $Q$-clouds resemble BPS Skyrmions in certain aspects, and correspond to universal non-perturbative solutions in (complex) $|\Phi|^{4}$ theory. In particular, we show that also $Q$-cloud solutions have negative $D$-terms. Our findings do not prove that $D$-terms must always be negative. But they indicate that it is unlikely to realize a positive $D$-term in a consistent physical system.
\end{abstract}

Keywords: energy momentum tensor, $Q$-ball, soliton, stability, $D$-term

\section{Introduction}

The $D$-term [1] is a particle property as fundamental as mass or spin, yet not known for any particle. It is defined through form factors of the energy momentum tensor [2]. As the electric form factor provides information on the charge distribution [3], so does the form factor associated with the $D$-term give insights into the distribution of internal forces inside a particle (and the interpretation is subject to the same type of limitations) [4].

Information on the $D$-term can be accessed in hard exclusive reactions [5, $6,7]$. Theoretical studies dedicated to the $D$-term include soft pion theorems, chiral perturbation theory, lattice QCD, soliton models, nuclear models, bag

Preprint submitted to Nuclear Physics A

April 18, 2016

(C) 2016. This manuscript version is made available under the Elsevier user license http://www.elsevier.com/open-access/userlicense/1.0/ 
and spectator models, and dispersion techniques $[1,4,8,9,10,11,12,13,14$, $15,16,17,18,19,20,21,22]$. Remarkably, in all theoretical studies so far the $D$-terms (of pions, nucleons, nuclei, photons, $Q$-balls) were found to be negative.

In early works a connection was suspected between the sign of the $D$-term and the stability of a particle [10]. However, insightful studies in the $Q$-ball system [21, 22] revealed that meta-stable, unstable and excited solutions (which all still correspond to local minima of the action) have also negative $D$-terms.

An emerging question that motivates our study is whether the D-term can be positive in a physical system. To address this question, we will use the $Q$-ball system as a theoretical laboratory once more, and investigate a particular limit in which unstable solutions "dissociate" into a "cloud" of free quanta.

$Q$-balls are non-topological solitons in theories with global symmetries [23, 24, 25]. They may have been created under the conditions of the early universe, were discussed as dark matter candidates, and have applications in astrophysics, cosmology, and particle physics $[26,27,28,29,30,31,32,33,34,35,36,37,38$, 39, 40, 41, 42, 43, 44, 45, 46].

Studies of the parametric limit in which unstable $Q$-balls delocalize, spread out over all space, and eventually form an infinitely dilute system of free quanta, date back to [27] where the interpretation as a " $Q$-cloud" was given.

$Q$-clouds are extreme, unstable field configurations. In contrast to unstable $Q$-balls or excited $Q$-ball states shown to have negative $D$-terms $[21,22]$ and decaying in several lighter stable $Q$-balls of the same total charge, $Q$-clouds are even energetically unstable against the tunneling to plane waves.

$Q$-ball solutions have been subject to modest interest in literature so far. Interesting recent developments are the demonstration of the existence of $Q$ clouds around Kerr black holes leading to the discovery of a new family of hairy black holes [47], and a possible connection to sphalerons [48]. Noteworthy is also the possibility to realize experimentally $Q$-cloud type configurations in ultra-cold Bose gases [49]. The modest attention $Q$-balls have received so far is perhaps related to their extreme instability. However, precisely this property makes $Q$-balls an ideal theoretical testing ground for the purposes of our work. Could such an extreme and unstable system exhibit a positive $D$-term?

In this work, we consider a scalar theory with $\mathrm{U}(1)$ symmetry and potential $V$ which admits solitons of the form $\Phi(\vec{r}, t)=\phi(r) e^{i \omega t}$ for $\omega_{\min }<\omega<\omega_{\max }$ with limiting frequencies fixed in terms of the potential. For $\omega$ approaching $\omega_{\min }$ one deals with stable $Q$-balls which are characterized by a constant charge density [24] and share many characteristics of fluid drops [21].

In the opposite limit $\omega \rightarrow \omega_{\max }$ the solutions are unstable. Their mass $M$ approaches from above $m Q$, where $Q$ is the charge of the solutions and $m$ denotes the mass of the elementary quanta [27]. Some properties of the solutions as $\omega$ approaches $\omega_{\max }$ were studied in [21]. But many questions remain open.

What are the behavior and the properties of the solutions as $\omega \rightarrow \omega_{\max }$ ? Does a well-defined limiting solution exist? And, to iterate the central question that motivates this work: considering that the dissociation of $Q$-matter into a $Q$-cloud constitutes a genuine instability, could one encounter in this system a positive $D$-term? The purpose of this work is to address these questions. 
The outline is as follows. In Sec. 2 we review $Q$-balls and their properties. In Sec. 3 we use the Newtonian interpretation of the equations of motion to establish qualitative expectations in the limit $\omega \rightarrow \omega_{\max }$. In Sec. 4 we demonstrate the existence of a limiting solution in terms of appropriately defined scaled coordinates and fields. The Secs. 5 and 6 are dedicated to the study of local and

global properties as $\omega$ approaches $\omega_{\max }$. In Sec. 7 we discuss the properties of the limiting solution, and interpret the results. Conclusions are presented in Sec. 8. Technical details are addressed in Appendices.

\section{The $Q$-ball system}

We study the complex scalar field theory defined in terms of the Lagrangian [24] with an effective (non-renormalizable) sixtic potential

$$
\begin{aligned}
& \mathcal{L}=\frac{1}{2}\left(\partial_{\mu} \Phi^{*}\right)\left(\partial^{\mu} \Phi\right)-V, \\
& V=A\left(\Phi^{*} \Phi\right)-B\left(\Phi^{*} \Phi\right)^{2}+C\left(\Phi^{*} \Phi\right)^{3} .
\end{aligned}
$$

The positive constants $A, B, C$ are such that

$$
0<\xi<1, \quad \xi=\frac{B^{2}}{4 A C} \equiv \frac{1}{2 \alpha},
$$

which guarantees that $V \geq 0 \forall \Phi$, and $V>0$ if $\Phi \neq 0$. We define in (3) the constant $\alpha$ for later convenience.

Non-topological solitons exist due to the global U(1) symmetry $\left(\Phi \rightarrow \Phi e^{i \eta}\right.$, $\left.\Phi^{*} \rightarrow \Phi^{*} e^{-i \eta}, \eta \in \mathbb{R}\right)$ of $\mathcal{L}$. In the soliton rest frame the solutions are given by

$$
\Phi(t, \vec{x})=\exp (i \omega t) \phi(r), \quad r=|\vec{x}|,
$$

where $\omega>0$ can be chosen without loss of generality, and $\phi(r)$ satisfies the following equation and boundary conditions (the primes denote differentiations with respect to the respective arguments)

$$
\begin{aligned}
& \phi^{\prime \prime}(r)+\frac{2}{r} \phi^{\prime}(r)+\omega^{2} \phi(r)-V^{\prime}(\phi)=0, \\
& \phi(0) \equiv \phi_{0}=\mathrm{const}, \phi^{\prime}(0)=0, \\
& \phi(r) \rightarrow 0 \text { for } r \rightarrow \infty
\end{aligned}
$$

Finite energy solutions exist for $\omega$ in the range [24]

$$
\begin{aligned}
& \omega_{\min }<\omega<\omega_{\max }, \\
& \omega_{\min }^{2}=\min _{\phi}\left[\frac{2 V(\phi)}{\phi^{2}}\right]=2 A(1-\xi)>0, \\
& \omega_{\max }^{2}=\left.V^{\prime \prime}(\phi)\right|_{\phi=0}=2 A .
\end{aligned}
$$


Notice that $\omega_{\max }$ defines the mass $m$ of the elementary quanta of the field $\Phi$ as

$$
m=\omega_{\max }=\sqrt{2 A} .
$$

The solutions can be classified as sketched in Fig. 1.

For $\omega_{\min }<\omega<\omega_{\text {abs }}$ one encounters stable solutions satisfying the absolute stability condition $M<m Q$ [28], where $M$ and $Q$ denote the soliton mass and charge. The term " $Q$-ball" was originally coined to denote solutions in the limit of $\omega$ approaching $\omega_{\min }[24]$.

For $\omega_{\text {abs }}<\omega<\omega_{c}$ the solutions are meta-stable, i.e. stable with respect to small fluctuations $[23,28]$. They satisfy a weaker "classical stability condition" which can be expressed, for instance, as $Q^{\prime}(\omega) \leq 0$. At the critical frequency $\omega_{c}$ the charge becomes minimal.

For $\omega_{c}<\omega<\omega_{\max }$ the unstable solutions can decay into stable $Q$-balls with the same charge but a lower total mass. In the limit $\omega \rightarrow \omega_{\max }$ one then deals with $Q$-clouds [27].

To conduct our study we will investigate properties related to the charge and energy-momentum tensor $T^{\mu \nu}$ (EMT), which are introduced in the following.

The conserved charge due to the $\mathrm{U}(1)$ symmetry is

$$
Q=\int \mathrm{d}^{3} r \rho_{\mathrm{ch}}(r), \quad \rho_{\mathrm{ch}}(r)=\omega \phi(r)^{2} .
$$

The canonical EMT of the theory (1) is symmetric, and static for the solutions (4) [21]. The energy density

$$
T_{00}(r)=\frac{1}{2} \omega^{2} \phi(r)^{2}+\frac{1}{2} \phi^{\prime}(r)^{2}+V(\phi),
$$

defines the mass $M=\int \mathrm{d}^{3} r T_{00}$. One finds $T_{0 k}=0$, i.e. our solutions have spin zero (see [36] for a discussion of spinning solutions). The spatial components

$$
T_{i j}=\left(\frac{r_{i} r_{j}}{r^{2}}-\frac{1}{3} \delta_{i j}\right) s(r)+\delta_{i j} p(r)
$$

define the stress tensor where $s(r)$ and $p(r)$ denote the distributions of shear

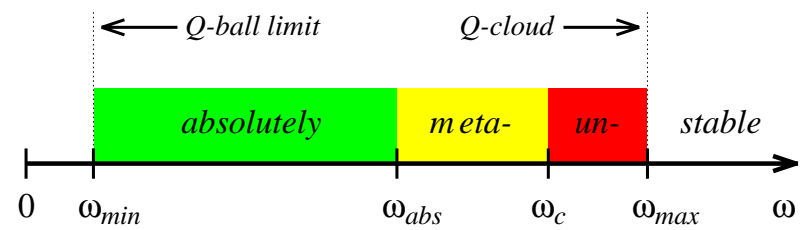

Figure 1: The solitons of the theory (1) are absolutely stable for $\omega_{\min }<\omega<\omega_{\text {abs }}$, classically stable for $\omega_{\text {abs }}<\omega<\omega_{c}$, and unstable for $\omega_{c}<\omega<\omega_{\max }$. The arrows indicate the $Q$-ball limit [24] and the $Q$-cloud limit [27]. The numerical values of the $\omega_{i}$ depend on the parameters of the theory in Eq. (2). 
forces and pressure given by

$$
\begin{aligned}
& s(r)=\phi^{\prime}(r)^{2} \\
& p(r)=\frac{1}{2} \omega^{2} \phi(r)^{2}-\frac{1}{6} \phi^{\prime}(r)^{2}-V(\phi) .
\end{aligned}
$$

The conservation of the EMT dictates that $s(r)$ and $p(r)$ are connected by the relation [4]

$$
\frac{2}{r} s(r)+\frac{2}{3} s^{\prime}(r)+p^{\prime}(r)=0,
$$

and $p(r)$ must obey [10] the von Laue condition [50], a necessary condition for stability,

$$
\int_{0}^{\infty} \mathrm{d} r r^{2} p(r)=0
$$

The constant $d_{1}$, to which we refer here as the $D$-term, completes the information content of the EMT and is given in terms of $s(r)$ or $p(r)$ as [4]

$$
\begin{aligned}
d_{1} & =5 \pi M \int_{0}^{\infty} \mathrm{d} r r^{4} p(r), \\
& =-\frac{4 \pi}{3} M \int_{0}^{\infty} \mathrm{d} r r^{4} s(r) .
\end{aligned}
$$

Other quantities of interest are "surface tension" $\gamma$, mean square radius $\left\langle r_{s}^{2}\right\rangle$ of the shear forces,

$$
\gamma=\int_{0}^{\infty} \mathrm{d} r s(r), \quad\left\langle r_{s}^{2}\right\rangle=\frac{1}{\gamma} \int_{0}^{\infty} \mathrm{d} r r^{2} s(r),
$$

and mean square radii of the energy and charge densities

$$
\left\langle r_{E}^{2}\right\rangle=\frac{\int \mathrm{d}^{3} r r^{2} T_{00}(r)}{\int \mathrm{d}^{3} r T_{00}(r)},\left\langle r_{Q}^{2}\right\rangle=\frac{\int \mathrm{d}^{3} r r^{2} \rho_{\mathrm{ch}}(r)}{\int \mathrm{d}^{3} r \rho_{\mathrm{ch}}(r)} .
$$

From Eqs. $(5,6)$ one deduces that the $\phi(r)$ behave as

$$
\begin{aligned}
& \phi(r)=\phi_{0}+\left(V^{\prime}\left(\phi_{0}\right)-\omega^{2} \phi_{0}\right) \frac{r^{2}}{6}+\ldots \text { small } r, \\
& \phi(r)=\frac{c_{\infty}}{r} \exp \left(-r \sqrt{\omega_{\max }^{2}-\omega^{2}}\right)+\ldots \text { large } r,
\end{aligned}
$$

where the dots indicate subleading terms, and $\phi_{0}$ and $c_{\infty}$ follow from solving the boundary value problem $(5,6)$.

When presenting numerical results we use the same parameters and $\omega$-range as in Ref. [21],

$$
\begin{gathered}
A=1.1, \quad B=2.0, \quad C=1.0, \\
\omega_{\min }^{2}=0.2, \quad \omega_{\max }^{2}=2.2 .
\end{gathered}
$$

With these parameters the other frequencies in Fig. 1 take the values $\omega_{\text {abs }}^{2} \approx 1.55$ and $\omega_{\mathrm{c}}^{2} \approx 1.9[21]$. 


\section{Towards the $Q$-cloud limit}

We first establish qualitative expectations in the limit $\omega \rightarrow \omega_{\max }$. For that we explore the analogy of (5) to a Newtonian equation for the 1D motion of a unit mass particle described by the coordinate $z(t)$, which is subject to a timeand velocity-dependent frictional force $F_{\text {fric }}(\dot{z}, t)$ in an effective potential $U_{\text {eff }}(z)$ defined as

$$
\begin{aligned}
& \ddot{z}(t)=F_{\text {fric }}(\dot{z}, t)-\nabla U_{\text {eff }}(z) \\
& F_{\text {fric }}(\dot{z}, t)=-\frac{2}{t} \dot{z}(t), \quad U_{\text {eff }}(z)=\frac{1}{2} \omega^{2} z^{2}-V(z),
\end{aligned}
$$

where we identify $r \leftrightarrow t$ and $\phi(r) \leftrightarrow z(t)$. At $t=0$ the particle starts with zero velocity (analog to $\phi^{\prime}(0)=0$ ) from the position $z_{0}$ (analog to $\phi_{0}$ ) chosen such that the particle will "slide down" in the potential $U_{\text {eff }}(z)$ shown in Fig. 2 and, after infinitely long time, stop at the origin (analog to $\phi(r) \rightarrow 0$ for $r \rightarrow \infty$ ).

This very fruitful analogy was used in [24] to illustrate the existence of the solutions. Only if $\omega>\omega_{\min }$ can solutions exist, since then there is a starting point $z_{0}>0$ where the particle has a potential energy $U_{\text {eff }}\left(z_{0}\right)>0$ so it can overcome the friction and make it to the origin. As long as $\omega<\omega_{\max }$ the potential $U_{\text {eff }}(z)$ will also dip below zero somewhere between $z_{0}$ and the origin, which is necessary to allow the particle to dissipate its initial energy before arriving at the origin.

When $\omega$ is close to $\omega_{\min }$ one deals with " $Q$-balls," i.e. solutions which exhibit extended plateaus in the inner region with nearly constant charge density [24] and resemble fluid drops in many aspects [21].

Here we are interested in the opposite limit $\omega \rightarrow \omega_{\max }$. In this regime the region of $z$ where $U_{\text {eff }}(z)<0$ shrinks, causing the initial positions $z_{0}$ (from which the particle has to be released to arrive at the origin with zero velocity) to decrease rapidly, see Fig. 2.

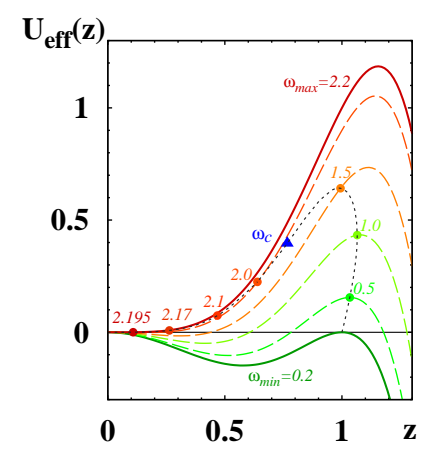

Figure 2: $U_{\text {eff }}(z)=\frac{1}{2} \omega^{2} z^{2}-V(z)$ as function of $z$ for the limiting values $\omega_{\min }^{2}=0.2$ and $\omega_{\max }^{2}=2.2$ (solid lines), and for the selected values $\omega^{2}=0.5,1.0,1.5,2.0$ (dashed lines). The circles show the initial values $z_{0}$ for each $\omega^{2}$, which lie on a continuous (dotted) curve [21]. The starting points $z_{0}$ for $\omega_{c}^{2} \simeq 1.9$ and $\omega^{2}=2.1,2.17,2.195$ are indicated, without plotting $U_{\text {eff }}(z)$ for these values. 
In the language of fields, this means the magnitudes of $\phi(r)$ and hence the charge and energy densities decrease. At the same time, the fields decay at large $r$ more and more slowly according to (21), implying that the spatial extension of the field configurations grows. The decrease of charge and energy densities is overall overwhelmed by the growth of the spatial extension of the solutions. As a consequence the total charge and mass diverge.

The patterns how charge, mass and size of the solutions diverge were studied numerically in [21]. To make definite statements, it is convenient to define

$$
\varepsilon=\sqrt{\omega_{\max }^{2}-\omega^{2}}>0 .
$$

It was found that $M, Q$, and mean radii diverge like $1 / \varepsilon$, and $d_{1} \propto 1 / \varepsilon^{2}$, while the surface tension $\gamma \propto \varepsilon^{3}[21]$.

As $\omega^{2}$ reaches $\omega_{\max }^{2}$ the effective potential $U_{\text {eff }}(z)$ never dips below zero. At first glance, the only viable solution seems to be when the particle is placed at $z_{0}=0$ which corresponds to a trivial vacuum solution $\phi(r)=0 \forall r$. There is, however, also a non-trivial "critical" solution, which is best seen in terms of adequately scaled units.

\section{Rescaling \& existence of limit}

To study the $Q$-cloud limit rigorously we introduce dimensionless coordinates $\vec{x} \rightarrow \varepsilon \vec{r}$ with $x=|\vec{x}|=\varepsilon r$, and define dimensionless rescaled fields $\tilde{\varphi}(x)$ as

$$
\phi(r)=\varepsilon \frac{\tilde{\varphi}(x)}{\sqrt{B}}, \quad x=\varepsilon r .
$$

For later convenience we introduce $1 / \sqrt{B}$ in (26) with the positive, dimensionless parameter $B$ of the potential (2). We also define the dimensionless quantity

$$
\tilde{\varepsilon}^{2}=\frac{\varepsilon^{2}}{m^{2}} \equiv \frac{\varepsilon^{2}}{2 A}, \quad 0<\tilde{\varepsilon}^{2}<\xi,
$$

whose range follows from (7). In terms of the rescaled coordinates and fields, the equation of motion becomes

$$
\begin{aligned}
& \tilde{\varphi}^{\prime \prime}(x)+\frac{2}{x} \tilde{\varphi}^{\prime}(x)-\tilde{\varphi}(x)+4 \tilde{\varphi}^{3}(x)-6 \alpha \tilde{\varepsilon}^{2} \tilde{\varphi}^{5}(x)=0, \\
& \tilde{\varphi}(0)=\mathrm{const}, \quad \tilde{\varphi}^{\prime}(0)=0, \quad \tilde{\varphi}(x) \rightarrow 0 \text { as } x \rightarrow \infty,
\end{aligned}
$$

with the parameter $\alpha=2 A C / B^{2}$ as defined in Eq. (3).

At respectively small and large $x$ the rescaled fields behave as

$$
\begin{aligned}
& \tilde{\varphi}(x)=\tilde{\varphi}_{0}+\left(\tilde{\varphi}_{0}-4 \tilde{\varphi}_{0}^{3}+6 \alpha \tilde{\varepsilon}^{2} \tilde{\varphi}_{0}^{5}\right) \frac{x^{2}}{6}+\mathcal{O}\left(x^{4}\right), \\
& \tilde{\varphi}(x)=\tilde{c}_{\infty} \frac{e^{-x}}{x} .
\end{aligned}
$$


The 'rescaled problem' (28) can be solved numerically for arbitrarily small $\varepsilon$. In Fig. 3a we show the solutions for selected values in the range $10^{-6} \leq \varepsilon \leq 10^{-2}$ for $\phi(r)$ as functions of $r$, i.e. in the 'usual' units restored according to (26). We include the result for $\varepsilon \approx 0.071$ corresponding to $\omega^{2}=2.195$ which was the $\omega$-value closest to $\omega_{\max }$ numerically tractable in [21].

Fig. 3a demonstrates how strongly the magnitudes of the solutions decrease with decreasing $\varepsilon$, and at the same time how much the solutions spread out.

In Fig. $3 \mathrm{~b}$ we plot the solutions in terms of rescaled fields and coordinates (26). The rescaled solutions cannot be distinguished from each other for $10^{-6} \leq$ $\varepsilon \lesssim 10^{-1}$ within the resolution of the plot. Deviations from the curves in Fig. $3 \mathrm{~b}$ start to be noticeable only for $\varepsilon \gtrsim 0.2$, as we will investigate in detail in Sec. 5 .

We remark that the rescaled problem (28) allows one to extend the study by orders of magnitude into the small- $\varepsilon$ region, which is restricted only by numerical accuracy. In our case the relative accuracy is typically in the range $10^{-8}-10^{-6}$. We know this from monitoring the numerical quality of the solutions by checking, e.g., that (15) holds within numerical accuracy, that the different representations $(16,17)$ yield the same value for $d_{1}$, and by performing other quality tests; see [21] for more details. Hence, our numerical method is not sufficiently accurate for $\varepsilon<10^{-6}$. But as is apparent from Fig. 3b, it is not necessary to go to such small values of $\varepsilon$.

The physical problem requires $\omega_{\min }^{2}<\omega^{2}<\omega_{\max }^{2}$ which implies $\tilde{\varepsilon}>0$, see Eqs. $(7,27)$. But in the rescaled problem (28) nothing prevents us from setting $\tilde{\varepsilon}=0$. This must be understood as a careful limiting procedure, otherwise the rescaling (26) would become singular.

Nevertheless, in the limit $\tilde{\varepsilon}=0$ the problem (28) has a regular solution $\tilde{\varphi}(x)$ which we have included in Fig. 3b, and which also cannot be distinguished from the curves plotted in Fig. $3 \mathrm{~b}$ which refer to finite $\varepsilon$ in the range $10^{-6} \leq \varepsilon \lesssim 0.1$. This shows how smoothly the limit is approached, and how numerically close to it the results are for finite $\varepsilon \lesssim 0.1$.
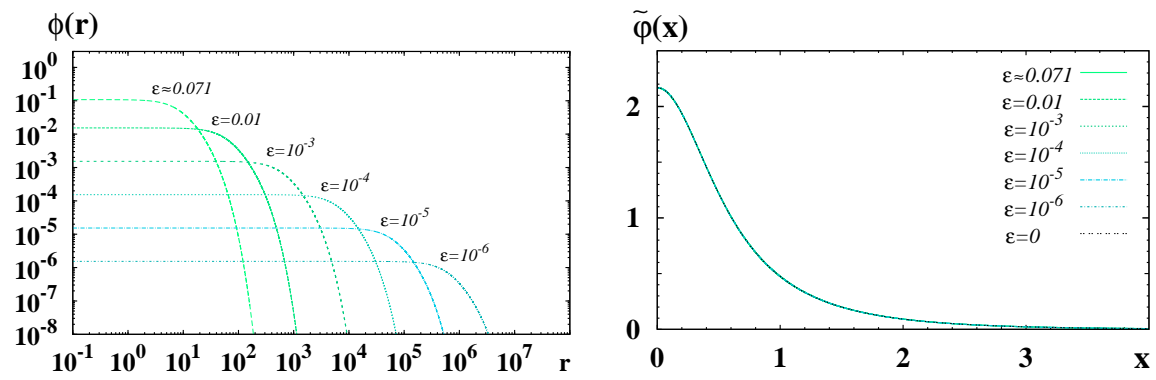

Figure 3: (a) The solutions $\phi(r)$ as functions of $r$ for different $\varepsilon$ in a double-logarithmic plot to illustrate how strongly the solutions decrease with $\varepsilon$ and at the same time spread out. The result for $\varepsilon \approx 0.071\left(\omega^{2}=2.195\right)$ was the value closest to the $Q$-cloud limit solved in [21] without the rescaling (26). (b) Solutions $\tilde{\varphi}(x)$ from (a) rescaled according to (26) vs. $x$. Also the limiting value $\varepsilon=0$ is shown. The different results are indistinguishable within the resolution of this plot. 


\section{Densities in the limit $\varepsilon \rightarrow 0$}

To investigate the behavior and properties of the solutions in the limit $\varepsilon \rightarrow 0$, one has to go to more sizable values of $\varepsilon>0.1$. In the following, in order to visualize how the rescaled fields $\tilde{\varphi}(x)$ approach the limiting case, we will present results for $\varepsilon=0,0.1,0.2,0.3,0.4,0.5$.

In Fig. 4a we show the rescaled fields $\tilde{\varphi}(x)$ as functions of $x$ for different $\varepsilon$. Despite this sizable $\varepsilon$-range, the variation of $\tilde{\varphi}(x)$ with decreasing $\tilde{\varepsilon}$ is modest. As the limit is approached, the solutions tend to grow in the center region and decrease at larger $x$. As a result, the limiting field is more strongly localized around $x=0$ than the solutions for finite $\varepsilon$.

To discuss further properties, we define the rescaled densities as follows (see App. Appendix A for alternative notation)

$$
\begin{aligned}
\rho_{\mathrm{ch}}(r) & =\varepsilon^{2} \frac{\omega}{B} \tilde{\rho}_{\mathrm{ch}}(x), & p(r) & =\frac{\varepsilon^{4}}{B} \tilde{p}(x), \\
T_{00}(r) & =\varepsilon^{2} \frac{2 A}{B} \tilde{T}_{00}(x), & s(r) & =\frac{\varepsilon^{4}}{B} \tilde{s}(x),
\end{aligned}
$$

with

$$
\begin{array}{rlrl}
\tilde{\rho}_{\mathrm{ch}}(x) & =\tilde{\varphi}(x)^{2}, \\
\tilde{T}_{00}(x) & =\tilde{\varphi}(x)^{2}+\tilde{\varepsilon}^{2}\left[\frac{1}{2} \tilde{\varphi}^{\prime}(x)^{2}-\frac{1}{2} \tilde{\varphi}(x)^{2}-\tilde{\varphi}(x)^{4}+\alpha \tilde{\varepsilon}^{2} \tilde{\varphi}(x)^{6}\right], \\
\tilde{p}(x) & = & -\frac{1}{6} \tilde{\varphi}^{\prime}(x)^{2}-\frac{1}{2} \tilde{\varphi}(x)^{2}+\tilde{\varphi}(x)^{4}-\alpha \tilde{\varepsilon}^{2} \tilde{\varphi}(x)^{6}, \\
\tilde{s}(x) & = & \tilde{\varphi}^{\prime}(x)^{2} .
\end{array}
$$

The rescaling is such that physical dimensions, including leading powers of $\varepsilon$, are stripped off in (30), such that the tilde-densities in Eq. (31) are expressed solely in terms of dimensionless parameters and fields $\alpha, \tilde{\varepsilon}, \tilde{\varphi}(x)$.

Interestingly, in the limiting case, and only in this case, the rescaled charge and energy densities coincide. The differential equation (14) connecting $p(r)$ and $s(r)$ holds analogously for $\tilde{s}(x)$ and $\tilde{p}(x)$, and is satisfied for $\tilde{\varepsilon}>0$ as well
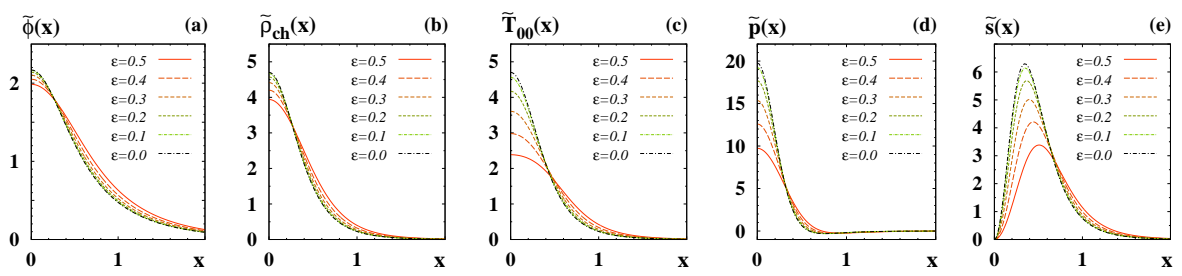

Figure 4: The fields $\tilde{\varphi}(x)$, charge densities $\tilde{\rho}_{\mathrm{ch}}(x)$, energy densities $\tilde{T}_{00}(x)$, pressure distributions $\tilde{p}(x)$, shear force distributions $\tilde{s}(x)$ vs. $x$ for selected values of $\varepsilon$. The fields and densities are dimensionless after the respective rescaling procedures $(26,30)$. 
as in the limit $\tilde{\varepsilon}=0$. The same holds true for the von Laue condition (15). We will follow up on the meaning of these observations in Sec. 7 .

In Fig. 4b and Fig. 4c we show the rescaled charge and energy densities. Both densities grow with decreasing $\varepsilon$ in the center region, decrease in the outer region, and coincide for $\varepsilon \rightarrow 0$. But $\tilde{T}_{00}(x)$ shows a stronger dependence on $\varepsilon>0$ than $\tilde{\rho}_{\text {ch }}(x)$ because, in contrast to charge density, the energy density encodes the full information on the dynamics of the theory.

The rescaled pressure function $\tilde{p}(x)$ is shown in Fig. 4d. As the limit is approached, $\tilde{p}(x)$ grows in the center and decreases in the outer region. For all solutions, including the limiting case, the rescaled pressure exhibits precisely one zero, as all ground state $Q$-ball solutions do [21].

In Fig. 4e we show the rescaled shear force distribution. In the opposite limit $\omega \rightarrow \omega_{\min }$ where $Q$-balls share some features of liquid drops [21], the shear force distributions approach the shape of a $\delta$-function concentrated around the "sharp edge" of the $Q$-balls. In the $Q$-cloud limit $\omega \rightarrow \omega_{\max }$ the positions of the peaks of $\tilde{s}(x)$ still indicate the size of the solutions. But the considerable widths of the $\tilde{s}(x)$ imply that the solutions have no "sharp edge" (not even in the rescaled coordinates $x$ ) but are diffuse.

In Fig. 5 we plot the results for $x^{2} \tilde{p}(x), x^{4} \tilde{p}(x)$ which shows more clearly the zeros of the pressure distribution. The results for $x^{2} \tilde{p}(x)$ in Fig. 5a illustrate the balance of the internal forces. Positive (negative) pressure in center (outer) region corresponds to repulsion (attraction). Repulsive and attractive forces balance each other exactly according to (15). The areas under the curves in Fig. 5a are equal within numerical accuracy for all $\varepsilon$.

The results for $x^{4} \tilde{p}(x)$ in Fig. 5b visualize the integrand of $d_{1}$ in (16). Since $x^{2} \tilde{p}(x)$ integrates to zero, the integral of $x^{4} \tilde{p}(x)$ is negative as the additional weight $x^{2}$ diminishes the contribution from the positive inner region and enhances that of the negative outer region. The same pattern was observed in other models, and illustrates the relation of $d_{1}$ to internal forces in the system.

This shows that the $D$-term of $Q$-clouds is negative. The sign of $d_{1}$ can be concluded independently and much more directly by exploring Eq. (17) which relates $d_{1}$ to the positive definite shear forces (12). In consistent and correctly solved field-theories one can use both (16) and (17) to show that $d_{1}<0$. In
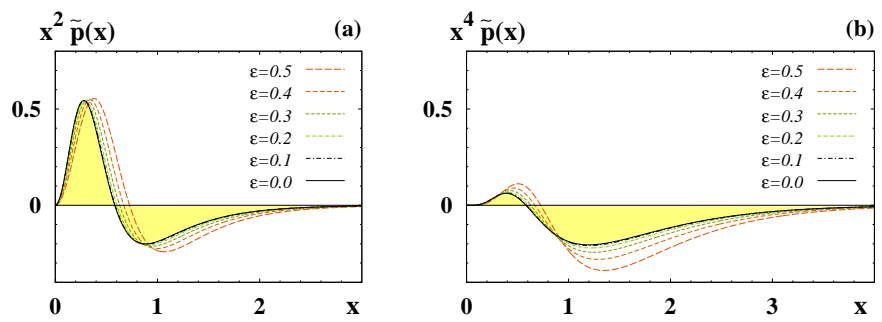

Figure 5: $\tilde{p}(x)$ weighted with $x^{2}$ and $x^{4}$ vs. $x$ for selected $\varepsilon$. Integrating $x^{2} \tilde{p}(x)$ yields zero, Eq. (15). Integrating $x^{4} \tilde{p}(x)$ yields a negative result, which explains that $d_{1}<0$, see Eq. (16). For $\varepsilon=0$ the areas under the curves are shaded. 
fact, for regular $Q$-ball solutions the relation (17) shows immediately — without numerical calculations - that $d_{1}<0[21,22]$. However, concluding the sign of $d_{1}$ from (16) allows one to verify the von Laue condition (15) which provides a cross check whether the equations of motion (here the minimization of the soliton energy) have been correctly solved. This is of particular importance in our case, since the limiting solution is strictly speaking singular and many properties, including the $D$-term, diverge such that one cannot take the equivalence of the representations (16) and (17) for $d_{1}$ for granted.

\section{Global properties for $\varepsilon \rightarrow 0$}

The global properties of the solutions, which follow from integrating the densities, can be expressed as (the notation is such that the left-hand sides in (32) have well-defined finite limits for $\varepsilon \rightarrow 0$ )

$$
\begin{aligned}
\varepsilon Q & =\frac{\omega}{B} \int \mathrm{d}^{3} x \tilde{\rho}_{\mathrm{ch}}(x), \\
\varepsilon M & =\frac{2 A}{B} \int \mathrm{d}^{3} x \tilde{T}_{00}(x), \\
\varepsilon^{2} d_{1} & =\frac{5(\varepsilon M)}{4 B} \int \mathrm{d}^{3} x x^{2} \tilde{p}(x)=-\frac{(\varepsilon M)}{3 B} \int \mathrm{d}^{3} x x^{2} \tilde{s}(x), \\
\varepsilon^{2}\left\langle r_{s}^{2}\right\rangle & =\frac{\int_{0}^{\infty} \mathrm{d} x x^{2} \tilde{s}(x)}{\int_{0}^{\infty} \mathrm{d} x \tilde{s}_{(}(x)} \\
\varepsilon^{2}\left\langle r_{Q}^{2}\right\rangle & =\frac{\int \mathrm{d}^{3} x x^{2} \tilde{\rho}_{\mathrm{ch}}(x)}{\int \mathrm{d}^{3} x \tilde{\rho}_{\mathrm{ch}}(x)} \\
\varepsilon^{2}\left\langle r_{E}^{2}\right\rangle & =\frac{\int \mathrm{d}^{3} x x^{2} \tilde{T}_{00}(x)}{\int \mathrm{d}^{3} x \tilde{T}_{00}(x)}, \\
\varepsilon^{-3} \gamma & =\frac{1}{B} \int_{0}^{\infty} \mathrm{d} x \tilde{s}(x) .
\end{aligned}
$$

From (32) we expect that with decreasing $\varepsilon$ the charge, mass and mean radii diverge as $1 / \varepsilon$ and $d_{1}$ grows as $1 / \varepsilon^{2}$, while the surface tension $\gamma$ vanishes as $\varepsilon^{3}$. Another relevant property is surface energy which scales as

$$
\varepsilon^{-1} E_{\text {surf }}=\frac{1}{B} \int \mathrm{d}^{3} x \tilde{s}(x) .
$$

where $E_{\text {surf }}$ can be defined in equivalent ways as [21] (here $D=3$ denotes the number of space dimensions),

$$
E_{\text {surf }}=4 \pi \gamma\left\langle r_{s}^{2}\right\rangle \equiv D(M-\omega Q)
$$

Thus $E_{\text {surf }} \propto \varepsilon$ for $\varepsilon \rightarrow 0$. We will need this result later.

We recall that for $\omega \rightarrow \omega_{\text {min }} Q$-balls develop a "sharp edge" which makes $\gamma$ and $E_{\text {surf }}$ well-defined notions [24]. It is interesting that in the opposite limit $\omega \rightarrow \omega_{\max }$ surface tension and surface energy become irrelevant. 

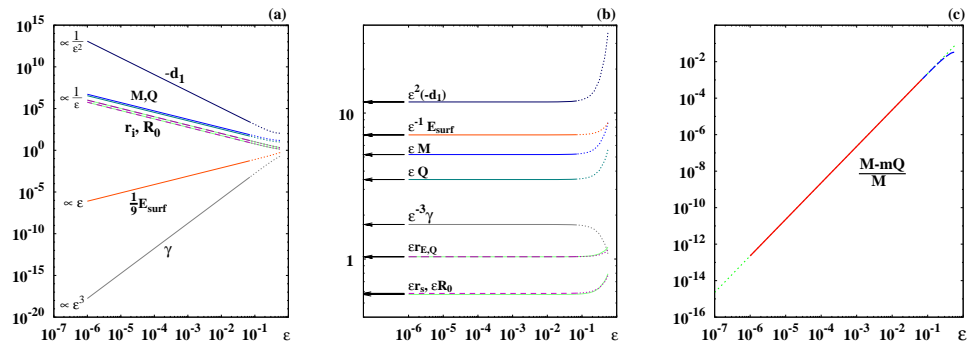

Figure 6: (a) $Q$-ball properties as functions of $\varepsilon$. From top to bottom: $d_{1}, M, Q, r_{i}=\left\langle r_{i}^{2}\right\rangle^{1 / 2}$ $(i=E, Q, s), R_{0}, E_{\text {surf }}, \gamma$. Dotted curves corresponding to $1.8 \leq \omega^{2} \leq 2.195$ are from [21], solid or long-dashed curves were obtained in this work. We plot $\frac{1}{9} E_{\text {surf }}$ to shift the curve and avoid intersections with other curves. (b) The same as (a) but for scaled properties. (c) $(M-m Q)$ normalized with respect to $M$ vs. $\varepsilon$. Solid (dashed) lines: results obtained here (in Ref. [21]) as described in caption of Fig. 6. Dotted line: the analytically calculated result from Eq. (34) with higher order terms $\mathcal{O}\left(\tilde{\varepsilon}^{4}\right)$ neglected.

In Fig. 6a we show numerical results for the properties $d_{1}, M, Q, r_{i}=\left\langle r_{i}^{2}\right\rangle^{1 / 2}$ $(i=E, Q, s), R_{0}, E_{\text {surf }}$ and $\gamma$. Here $R_{0}$ denotes the position of the zero of the pressure, i.e. $p\left(R_{0}\right)=0$. We include the results from [21] (dotted lines) for $1.8 \leq \omega^{2} \leq 2.195$ corresponding to $0.071 \lesssim \varepsilon \lesssim 0.63$. The results obtained here extend the work of [21] down to $\varepsilon=10^{-6}$ (solid lines). We stress that we obtain within numerical accuracy the same result for $d_{1}$ using Eq. (12) or (16).

On a plot with the resolution of Fig. 6a the curves for $\left\langle r_{E}^{2}\right\rangle^{1 / 2}$ and $\left\langle r_{Q}^{2}\right\rangle^{1 / 2}$ are practically on top of each other, which is due to the fact that both radii coincide for $\varepsilon \rightarrow 0$. In Fig. 6a also the curves for $\left\langle r_{s}^{2}\right\rangle^{1 / 2}$ and $R_{0}$ are practically indistinguishable, ${ }^{1}$ but this effect is accidental due to the logarithmic scale in the plot because $R_{0}$ and $\left\langle r_{s}^{2}\right\rangle^{1 / 2}$ are numerically close but not equal.

In Fig. $6 \mathrm{~b}$ we show the behavior of the adequately scaled quantities as defined on the left-hand sides in (32). The arrows indicate the results in the limiting case $\varepsilon=0$. The figure illustrates that the scaling regime practically sets in for $\varepsilon \lesssim 0.1$, i.e. it could just be observed in [21].

Next we discuss how $M$ approaches $m Q$ for $\varepsilon \rightarrow 0$. For $\omega>\omega_{c}$ (i.e. for $\varepsilon \lesssim 0.55$ for the parameters used in this work) one has $M>m Q$, see Sec. 2, such that $M$ approaches $m Q$ from above [27]. We can make a more quantitative statement by evaluating the leading term of $(M-m Q) / M$. Remarkably, the result can be computed analytically, see App. Appendix B,

$$
\frac{M-m Q}{M}=\frac{1}{2} \tilde{\varepsilon}^{2}+\mathcal{O}\left(\tilde{\varepsilon}^{4}\right) .
$$

In Fig. $6 \mathrm{c}$ we show $(M-m Q) / M$ down to $\varepsilon \geq 10^{-6}$ using the numerical results

\footnotetext{
${ }^{1}$ We use the occasion to correct 2 details in Fig. 11 of [21]. In that figure the results for $\left\langle r_{s}^{2}\right\rangle^{1 / 2}$ were reported to be on top of the curves for $\left\langle r_{E}^{2}\right\rangle^{1 / 2}$ and $\left\langle r_{Q}^{2}\right\rangle^{1 / 2}$, while they were actually on top of $R_{0}$ (as in this work). In addition, the shift of the curve for $E_{\text {surf }}$ by the factor $\frac{1}{9}$ was not mentioned in the caption of Fig. 11 of [21]. These corrections have no consequence on the results and conclusions of [21].
} 
for $M$ and $Q$ displayed in Fig. 6. We include the analytical result (34) neglecting higher order terms $\mathcal{O}\left(\tilde{\varepsilon}^{4}\right)$. In the regime $\varepsilon<10^{-2}$ the numerical results practically coincide with the leading order asymptotics of Eq. (34).

\section{Interpretation}

Having established the behavior of the properties of the solutions in the limit $\varepsilon \rightarrow 0$, we now turn to the interpretation of the results. One way to understand the limiting solution consists of interpreting it as a dissociation of the unstable solutions into an infinitely dilute system of uniformly distributed free $Q$-quanta, a "Q-cloud" [27].

That the system becomes dilute can be seen from the densities $(30,31)$. At every point in space the charge and energy densities vanish as $\varepsilon^{2}$, which shows that the system is "infinitely dilute." At the same time the internal forces characterized by $p(r)$ and $s(r)$ vanish even faster as $\varepsilon^{4}$. This implies that in the $Q$-cloud limit the interactions between the quanta decrease, and they become free. That in the limit the $Q$-quanta can be considered free may be inferred alternatively from the observation that in the limit the rescaled charge and energy densities become equal, see Eq. (31), implying that $M=m Q$. The conserved charge $Q$ "counts" the number of quanta. Thus, the total mass of the system is given by the number of elementary quanta multiplied by their mass $m$. This in turn implies that the binding energy vanishes, i.e. the quanta are free in the limit. (As the absolute stability condition $M<m Q$ is not satisfied for $\omega_{\mathrm{abs}}<\omega<\omega_{\max }$, the limit of a free gas of quanta is approached from above through the regimes of meta- and unstable solutions.) The last statement to clarify is the "uniform" distribution of the free quanta. This is explained by considering Fig. 3a. The solutions, and hence their charge or energy densities, are practically constant functions of $r$ up to distances of order $1 / \varepsilon$. For a sufficiently small $\varepsilon$ one could envision the "visible universe" filled with a uniformly distributed dilute gas of $Q$-quanta.

The results obtained in this work suggest also an alternative interpretation. For that let us first investigate how the relation of $M$ and $m Q$ arises. A powerful tool to provide insight in this respect is the virial theorem [29]. This theorem is derived exploring Eqs. $(9,10)$ which allow us to express the mass $M$, for a fixed charge $Q$, as

$$
M=\frac{1}{2} E_{\mathrm{ch}}+\frac{1}{2} E_{\mathrm{surf}}+E_{\mathrm{pot}}
$$

where (keeping the number of dimensions $D=3$ general)

$$
\begin{array}{cc}
E_{\text {surf }}=\int \mathrm{d}^{D} r \phi^{\prime}(r)^{2}, & E_{\text {pot }}=\int \mathrm{d}^{D} r V(\phi), \\
I=\int \mathrm{d}^{D} r \phi(r)^{2}, & E_{\mathrm{ch}}=\frac{Q^{2}}{I} .
\end{array}
$$

Now we define $M(\lambda)$ by evaluating (35) for dilatational variations of the solutions $\phi(r) \rightarrow \phi(\lambda r)$ with $\lambda>0$, and substituting $\vec{r} \rightarrow \lambda \vec{r}$ in the integrals in (36). 
We obtain

$$
M(\lambda)=\frac{1}{2} E_{\mathrm{ch}} \lambda^{D}+\frac{1}{2} E_{\mathrm{surf}} \lambda^{2-D}+E_{\mathrm{pot}} \lambda^{-D} .
$$

For $\lambda=1$ one has $M^{\prime}(\lambda)=0$ and $M^{\prime \prime}(\lambda)>0$ since, when setting $\lambda$ to unity, we restore the solutions $\phi(r)$ which minimize the energy functional. The statement $M^{\prime}(\lambda)=0$ at $\lambda=1$ is often referred to as virial theorem. From this relation one can derive the von Laue condition (15) [21]. Another usage of the virial theorem is to eliminate $E_{\text {pot }}$ in $M$ which yields the second expression for $E_{\text {surf }}$ in Eq. (33).

It is instructive to express $M(\lambda)$ in terms of rescaled fields (26). (Notice that for $\varepsilon \neq 0$ the substitutions $\vec{r} \rightarrow \lambda \vec{r}, \vec{r} \rightarrow \varepsilon \vec{x}$ commute with $\vec{r} \rightarrow \varepsilon \vec{x}, \vec{x} \rightarrow \lambda \vec{x}$.) Inserting $(30,31)$ in $(37)$ we obtain

$$
\begin{aligned}
\varepsilon M(\lambda) & =\frac{2 A}{B} \int \mathrm{d}^{D} x\left\{\frac{\lambda^{D}+\lambda^{-D}}{2} \tilde{\varphi}(x)^{2}\right. \\
& \left.+\tilde{\varepsilon}^{2}\left[\frac{\lambda^{2-D}}{2} \tilde{\varphi}^{\prime}(x)^{2}-\frac{\lambda^{D}}{2} \tilde{\varphi}(x)^{2}-\lambda^{-D} \tilde{\varphi}(x)^{4}\right]+\lambda^{-D} \alpha \tilde{\varepsilon}^{4} \tilde{\varphi}(x)^{6}\right\} .
\end{aligned}
$$

Setting $\lambda=1$ in (38) we recover the expression which follows directly from integrating $T_{00}(r)$ in $(30)$, namely

$$
\left.\varepsilon M(\lambda)\right|_{\lambda=1} \equiv \varepsilon M=\frac{2 A}{B} \int \mathrm{d}^{D} x \tilde{T}_{00}(x) .
$$

From $\varepsilon M^{\prime}(\lambda)$ at $\lambda=1$ we obtain the von Laue condition (15) formulated in terms of the rescaled pressure function

$$
\left.\varepsilon M^{\prime}(\lambda)\right|_{\lambda=1}=\frac{\varepsilon^{2} D}{B} \int \mathrm{d}^{D} x \tilde{p}(x)=0 .
$$

The expressions for $\varepsilon M(\lambda)$ and $\varepsilon M$ contain terms with explicit powers of $\tilde{\varepsilon}^{0}, \tilde{\varepsilon}^{2}, \tilde{\varepsilon}^{4}$. As $\tilde{\varepsilon}$ decreases the term $\propto \tilde{\varepsilon}^{0}$ dominates, and becomes the sole contribution to $\varepsilon M$ in the limit $\varepsilon \rightarrow 0$, where $\omega \rightarrow \omega_{\max }=m$ with the mass $m$ (8) of the elementary field $\Phi(x)$, such that we find

$$
\lim _{\varepsilon \rightarrow 0} \frac{M}{Q}=m
$$

meaning that the mass of the critical solution is entirely fixed in terms of its charge. This result can be deduced equivalently from $(30,31)$ or $(32 \mathrm{~h}, 34)$.

The virial theorem explains why the mass of the solution is fixed in terms of its charge: the term $\propto \varepsilon^{0}$ in (38) is the leading contribution in $\varepsilon M(\lambda)$, but drops out exactly from $\varepsilon M^{\prime}(\lambda)$ at $\lambda=1$. The question of how the solution acquires stability (in the sense of a local minimum of the action) is therefore answered by higher order terms in (38) which contain information on the dynamics of the 
theory (1). Thus, although they do not contribute to the mass of the critical solution, the subleading terms in (38), which encode the dynamics of the theory, determine the shape of the solution.

It is instructive to clarify also the relative importance of the subleading terms proportional to $\tilde{\varepsilon}^{2}$ and $\tilde{\varepsilon}^{4}$ in (38). The variational problem of minimizing $\varepsilon M(\lambda)$ receives information on the details of the theory, encoded in the dimensionless parameter $\alpha=2 A C / B^{2}$, only through the subsubleading term $\propto \tilde{\varepsilon}^{4}$ in (38).

In the limit, the terms $\propto \tilde{\varepsilon}^{2}$ determine the solution, while the term $\propto \tilde{\varepsilon}^{4}$ becomes irrelevant and drops out. Thus, the critical solution depends on the parameters $A, B$ of the theory (1), but not on $C$. Moreover, the dependence on $A, B$ is trivial in the sense that it provides trivial overall prefactors. This is evident also from the equation of motion which for $\varepsilon \rightarrow 0$ is given by

$$
\tilde{\varphi}^{\prime \prime}(x)+\frac{2}{x} \tilde{\varphi}^{\prime}(x)-\tilde{\varphi}(x)+4 \tilde{\varphi}^{3}(x)=0,
$$

with boundary conditions as specified in (28). This is a universal equation independent of the details of the theory (1) as encoded in the numerical values of the parameters $A, B, C$. Equation (42) is actually universal for all (complex) $|\Phi|^{4}$ theories with a negative coupling. (Notice that it was crucial to include the factor $B^{-1 / 2}$ in the rescaling of the fields (26) in order to obtain the "universal" equation of motion (42) in the limiting case.)

We use the term universality in this context to stress that instead of $C|\Phi|^{6}$ in the potential (2) we could have had started with any positive $C^{\prime}|\Phi|^{n}$ term with even $n \geq 8$. After the rescaling and limiting procedure, we would have arrived at the same universal equation (42) with no memory of powers beyond $|\Phi|^{4}$ in the potential (2).

At this point it is important to realize that, while in the limiting case there is no memory of it in the rescaled equation of motion, the power beyond $|\Phi|^{4}$ is crucial for providing proper boundary conditions for the theory (1). In fact, the potential (2) would be unbound from below without a positive term $|\Phi|^{n}$ with a power $n=6$ or higher. Thus, although the explicit dependence on the parameter $C$ drops out, it is crucial that the critical solution is understood as a careful limiting procedure with a positive higher order term.

With the term $\propto C|\Phi|^{6}$ becoming irrelevant for $\varepsilon \rightarrow 0$, the renormalizability of the theory (1) may seem restored. But this is a subtle issue for two reasons. First, we deal with classical soliton solutions and quantum corrections (whose consideration is beyond the scope of this work) have to be considered [51]. Second, a $|\Phi|^{4}$ theory with a negative coupling constant is actually ill-defined, as the potential is not bound from below. Thus, the limiting solution has to be understood within a careful limiting procedure with a however small nonrenormalizable term $\propto C|\Phi|^{n}>0$ in the potential with even $n=6$ or higher.

The limiting solution in the $Q$-ball system is reminiscent of the critical [52, $53]$ monopole solution $[54,55]$ in the Georgi-Glashow model $[56,57]$ in three characteristic respects. First, the mass of the critical solution is fixed in terms of its charge, a property arising from symmetries of the Lagrangian (1) rather than its dynamics (which, of course, determines the shape of the limiting solution). 
Second, it requires the presence of higher order terms in the original Lagrangian which become irrelevant in the limit and whose only role consists of providing boundary conditions for the theory. Third, from $(32 \mathrm{~h})$ we obtain the inequality $M \geq \omega Q$ which holds for all solutions, and becomes saturated in the limit

$\omega \rightarrow \omega_{\max }=m$. This imitates the saturation of a Bogomol'nyi-type bound for critical monopole solutions. Due to the simple U(1) symmetry, here the bound takes a somewhat simplistic form.

The observation that $Q$-clouds correspond to universal non-perturbative solutions in a complex $|\Phi|^{4}$ theory with negative coupling may have interesting implications. As the potential is not bound from below, taken by itself a $|\Phi|^{4}$ theory with a negative coupling is unphysical (unless supplemented by a however small positive, non-renormalizable higher order term, as discussed above). At the same time, it is connected by analytical continuation to a complex $|\Phi|^{4}$ theory with a positive coupling. Analytical continuation is at the heart of the proof that in general perturbative expansions have zero convergence radius [58]. A meaningful treatment of a theory is provided in the framework of "resurgent trans-series analysis" [59] where the perturbative series is combined with a series over all non-perturbative contributions, with the terms in both series connected to each other via specific "resurgence relations." A question emerging in this context is whether our universal non-perturbative solution in complex $|\Phi|^{4}$ theory (with negative coupling constant) could contribute to such a trans-series. This interesting question, which is beyond the scope of our study, could potentially shed new light on the non-perturbative sector of complex $|\Phi|^{4}$ theories.

Finally, let us remark that irrespective of its interpretation, the limiting solution constitutes a local extremum of the action. This is reflected by the fact that it satisfies the von Laue condition, which means that the internal forces balance each other exactly. This balance of forces is a necessary condition for stability, but not sufficient. In fact, the $Q$-cloud solution constitutes a highly unstable field configuration: the smallest disturbances would result in formation of small and stable $Q$-balls. This could make $Q$-clouds of interest as (toy) models for the inflationary era in the early universe, in particular if one succeeded in driving the limit $\varepsilon \rightarrow 0$ dynamically [60].

Worth mentioning in this context is the interesting connection of the $D$-term to the cosmological constant and inflation discussed in [61].

Although in line with results from all other theoretical systems, the negative $D$-term of the $Q$-cloud is still remarkable. If even such an extremely unstable system has a negative $D$-term, one may doubt whether it is possible to encounter a consistent system with a positive $D$-term. In this way our results contribute to the emerging understanding that $D$-terms are negative, which is rooted in the equilibrium of internal forces, even if it is a highly unstable equilibrium.

\section{Conclusions}

We have presented a study of soliton solutions in the $Q$-ball system [24] focusing on the limit where the frequency $\omega$ approaches its maximal value $\omega_{\max }$. 
This limit was studied previously in [27] where it was interpreted as the dissociation of unstable solutions into a dilute gas of free quanta, and in [21] where properties of the solutions were investigated and numerically found to exhibit characteristic scaling behavior, for example, mass $M \propto 1 / \varepsilon$ and $D$-term $\propto 1 / \varepsilon^{2}$ with $\varepsilon=\sqrt{\omega_{\max }^{2}-\omega^{2}}$. In this work, by working with adequately rescaled fields and coordinates, we were able to go far beyond what was numerically tractable in [21], and presented exact numerical solutions down to $\varepsilon \geq 10^{-6}$ confirming qualitative findings of [21]. We studied in detail the solution of the rescaled equations of motion in the $Q$-cloud limit $\varepsilon \rightarrow 0$ [27], and showed how smoothly this limit is approached. All properties of the limiting solution exhibit, after appropriate scaling, a smooth behavior as $\varepsilon \rightarrow 0$. We derived analytical results for rescaled quantities like $\varepsilon M$ expressed in terms of the limiting solution.

The limiting solution has fascinating properties. If we include in the rescaling the parameter $B$ of the potential $V=A \phi^{2}-B \phi^{4}+C \phi^{6}$, such that $\tilde{\varphi}(x)=\phi(r) /(\varepsilon \sqrt{B})$ with $x=\varepsilon r$, we obtain in the limiting case a "universal" soliton equation of motion for the rescaled field $\tilde{\varphi}(x)$ which is independent of the parameters $A, B, C$. More precisely, the parameters $A, B$ provide trivial prefactors for the properties, while $C$ drops out in the limit, i.e. the sixtic term in the potential $V(\phi)$ becomes "irrelevant" in the sense of critical phenomena.

We showed that the limiting solution shares features of critical monopoles, and observed that it corresponds to a universal non-perturbative solution in complex $|\Phi|^{4}$ theory with negative coupling, which may have implications for the non-perturbative sector of $|\Phi|^{4}$ theories. But the main feature of the limiting solution is that it is a highly unstable field configuration. The smallest disturbance would cause a decay into energetically favorable, small-size, stable $Q$-ball configurations. In this respect the $Q$-cloud resembles an undercooled gas, and could be of potential interest for (toy) models of the early universe.

Our initial motivation to study $Q$-clouds was triggered by the question whether such an extreme system with a genuine instability, could exhibit a positive $D$-term. But despite the extreme instability, the $Q$-cloud satisfies the von Laue condition. This means the internal forces balance each other exactly, although one deals with a highly unstable equilibrium situation. The balance of internal forces implies a negative sign for the $D$-term. Our study has shown that conclusions regarding the sign of $d_{1}$ of regular $Q$-balls [21] hold also for $Q$-clouds when viewed in terms of adequately scaled fields and coordinates. Considering the singularities associated with the $Q$-cloud limit, this result could not have been anticipated and required a careful and dedicated study.

As the $Q$-cloud constitutes the most unstable system we are aware of, the finding of a negative $D$-term in this extreme system is remarkable. Our work does not prove that all $D$-terms are negative. But considering that even such an extreme and unstable system as a $Q$-cloud has a negative $D$-term, it is difficult to imagine a consistent physical system with a positive $D$-term.

Our results support the emerging understanding that $D$-terms of particles are negative. It will be exciting to see whether this theoretical prediction will be confirmed for nucleons and atomic nuclei in experiments at Jefferson Lab, CERN or the future Electron-Ion Collider [62]. 
Acknowledgments. We thank Maxim Polyakov for discussions. The work was partly supported by the National Science Foundation under Contract No. 1406298.

\section{Appendix A. Systematic notation}

The notation introduced in Eqs. $(30,31)$ was very convenient for the discussion of the scaling behavior of the densities in Sec. 5. A notation showing more systematically the behavior of the charge density $\omega \rho_{\mathrm{ch}}(r)$ (where we include the factor of $\omega$ for convenience) and the EMT densities $T_{00}(r), p(r), s(r)$ is as follows

$$
\begin{aligned}
& \omega \rho_{\mathrm{ch}}(r)=\frac{(2 A)^{2}}{B}\left[\tilde{\varepsilon}^{2} \tilde{\varphi}(x)^{2}-\tilde{\varepsilon}^{4} \tilde{\varphi}(x)^{2}\right. \\
& T_{00}(r)=\frac{(2 A)^{2}}{B}\left[\tilde{\varepsilon}^{2} \tilde{\varphi}(x)^{2}+\tilde{\varepsilon}^{4}\left\{\frac{1}{2} \tilde{\varphi}^{\prime}(x)^{2}-\frac{1}{2} \tilde{\varphi}(x)^{2}-\tilde{\varphi}(x)^{4}\right\}+\tilde{\varepsilon}^{6} \alpha \tilde{\varphi}(x)^{6}\right], \\
& p(r)=\frac{(2 A)^{2}}{B}\left[\quad \tilde{\varepsilon}^{4}\left(-\frac{1}{6} \tilde{\varphi}^{\prime}(x)^{2}-\frac{1}{2} \tilde{\varphi}(x)^{2}+\tilde{\varphi}(x)^{4}\right)-\tilde{\varepsilon}^{6} \alpha \tilde{\varphi}(x)^{6}\right], \\
& s(r)=\frac{(2 A)^{2}}{B}\left[\quad \quad \tilde{\varepsilon}^{4} \tilde{\varphi}^{\prime}(x)^{2}\right.
\end{aligned}
$$

This shows that $\omega \rho_{\mathrm{ch}}(r), T_{00}(r), p(r), s(r)$ contain only even powers of $\tilde{\varepsilon}^{n}$ with $n=2,4,6$ with $\tilde{\varepsilon}^{2}=\varepsilon^{2} /(2 A)$ and the rescaled fields and coordinates as defined in (26). The curly brackets in Eq. (A.1b) highlight the contribution $\propto \tilde{\varepsilon}^{4}$ in $T_{00}(r)$ for later purposes.

The power $n=2$ is responsible for the leading (and in the limit sole) contributions to charge and energy densities $\omega \rho_{\mathrm{ch}}(r)$ and $T_{00}(r)$. The power $n=4$ provides the dominant (and in the limit sole) terms in the distributions of internal forces $s(r)$ and $p(r)$. The power $n=6$ accompanies the "irrelevant" (from the point of view of the limit) sixtic term from the potential, whose role is to provide proper boundary conditions for the (non-renormalizable) theory, as discussed in Sec. 7.

We remark that throughout this work we use $\varepsilon$ or $\tilde{\varepsilon}$ as defined in Eqs. $(25,27)$, depending which one yields a more convenient notation. At the expense of notational simplicity, we could have worked with $\tilde{\varepsilon}$ alone and preserved the dimensionality of fields and coordinates. 


\section{Appendix B. $M$ vs $m Q$ in the $Q$-cloud limit}

In this Appendix we show how $M$ approaches $m Q$, and derive the result in (34). This is of interest, as exact analytic results are rare in soliton models. We first prove that the expression highlighted by curly brackets in (A.1b) integrates to a higher order term in $\tilde{\varepsilon}$.

Working with rescaled coordinates and fields, we define the "equations of motion," $\operatorname{eom}(x)=0$, cf. Eq. (28), as

$$
\operatorname{eom}(x)=\tilde{\varphi}^{\prime \prime}(x)+\frac{2}{x} \tilde{\varphi}^{\prime}(x)-\tilde{\varphi}(x)+4 \tilde{\varphi}^{3}(x)-6 \alpha \tilde{\varepsilon}^{2} \tilde{\varphi}^{5}(x) .
$$

In this notation one can rewrite the term in the curly brackets in (A.1b) as follows

$$
\begin{array}{r}
x^{2}\left\{\frac{1}{2} \tilde{\varphi}^{\prime}(x)^{2}-\frac{1}{2} \tilde{\varphi}(x)^{2}-\tilde{\varphi}(x)^{4}\right\}=-x^{2} \operatorname{eom}(x) \tilde{\varphi}(x) \\
+3 x^{2} \tilde{p}(x)+\frac{\mathrm{d}}{\mathrm{d} x}\left[x^{2} \tilde{\varphi}(x) \tilde{\varphi}^{\prime}(x)\right]-3 \alpha \tilde{\varepsilon}^{2} x^{2} \tilde{\varphi}(x)^{6} .
\end{array}
$$

The term proportional to eom $(x)$ is zero anyway. Upon integration over $x$ the contribution of the pressure drops out due to the von Laue condition, Eqs. $(15,40)$, and so does the total derivative term. Thus, we find the identity

$$
\int \mathrm{d}^{3} x\left\{\frac{1}{2} \tilde{\varphi}^{\prime}(x)^{2}-\frac{1}{2} \tilde{\varphi}(x)^{2}-\tilde{\varphi}(x)^{4}\right\}=-3 \alpha \tilde{\varepsilon}^{2} \int \mathrm{d}^{3} x \tilde{\varphi}(x)^{6}
$$

which connects the subleading term in (A.1b) to the subsubleading term. In the limiting case $\tilde{\varepsilon} \rightarrow 0$, the right-hand-side of (B.2) yields exactly zero. In particular, we obtain for the mass

$$
\varepsilon M=\frac{2 A}{B} \int \mathrm{d}^{3} x\left(\tilde{\varphi}(x)^{2}-2 \alpha \tilde{\varepsilon}^{4} \tilde{\varphi}(x)^{6}\right) .
$$

The analog expression for $m Q$ is given by

$$
\varepsilon(m Q)=\frac{2 A}{B} \sqrt{1-\tilde{\varepsilon}^{2}} \int \mathrm{d}^{3} x \tilde{\varphi}(x)^{2} .
$$

In this way we obtain the exact result

$$
\frac{M-m Q}{M}=\frac{1-\sqrt{1-\tilde{\varepsilon}^{2}}-r \tilde{\varepsilon}^{4}}{1-r \tilde{\varepsilon}^{4}}, \quad r=2 \alpha \frac{\int \mathrm{d}^{3} x \tilde{\varphi}(x)^{6}}{\int \mathrm{d}^{3} x \tilde{\varphi}(x)^{2}},
$$

and expanding in a series in $\tilde{\varepsilon}$ we arrive at

$$
\frac{M-m Q}{M}=\frac{1}{2} \tilde{\varepsilon}^{2}+\left(\frac{1}{8}-r\right) \tilde{\varepsilon}^{4}+\mathcal{O}\left(\tilde{\varepsilon}^{6}\right) .
$$

We see that $M$ approaches $m Q$ from above, and find that the first term in the small- $\tilde{\varepsilon}$ expansion of the expression for $(M-m Q) / M$ can be evaluated 
analytically. Higher order terms in this expansion in general cannot be computed exactly, which we have indicated by quoting the exact coefficient of the $\tilde{\varepsilon}^{4}$-term in (B.6).

It is interesting to express Eq. (B.3) in terms of the original fields and coordinates, which yields

$$
M=\int \mathrm{d}^{3} r\left(2 A \phi(r)^{2}-2 C \phi(r)^{6}\right) .
$$

Thanks to the identity (B.1), the mass (of any solution) can be expressed in terms of two specific terms in the potential (2). We are not aware that this relation has been derived before in literature. From (B.7) we see that always $M \leq m^{2} \int \mathrm{d}^{3} r \phi(r)^{2}$, where the equal sign holds only in the $Q$-cloud limit when the contribution of $C \phi(r)^{6}$ becomes irrelevant.

\section{References}

\section{References}

[1] M. V. Polyakov and C. Weiss, Phys. Rev. D 60, 114017 (1999).

[2] H. R. Pagels, Phys. Rev. 144 (1965) 1250.

[3] R. G. Sachs, Phys. Rev. 126, 2256 (1962).

[4] M. V. Polyakov, Phys. Lett. B 555 (2003) 57.

[5] D. Müller et al., Fortsch. Phys. 42, 101 (1994). X. D. Ji, Phys. Rev. Lett. 78, 610 (1997); Phys. Rev. D 55, 7114 (1997). A. V. Radyushkin, Phys. Lett. B 380, 417 (1996); Phys. Lett. B 385, 333 (1996); Phys. Rev. D 56, 5524 (1997). J. C. Collins, L. Frankfurt and M. Strikman, Phys. Rev. D 56, 2982 (1997).

[6] X. D. Ji, J. Phys. G 24, 1181 (1998). A. V. Radyushkin, arXiv:hepph/0101225. K. Goeke, M. V. Polyakov and M. Vanderhaeghen, Prog. Part. Nucl. Phys. 47, 401 (2001). M. Diehl, Phys. Rept. 388 (2003) 41. A. V. Belitsky and A. V. Radyushkin, Phys. Rept. 418, 1 (2005). S. Boffi and B. Pasquini, Riv. Nuovo Cim. 30, 387 (2007).

[7] O. V. Teryaev, Phys. Lett. B 510, 125 (2001). A. V. Belitsky, D. Müller and A. Kirchner, Nucl. Phys. B 629, 323 (2002). A. V. Belitsky and D. Müller, Nucl. Phys. A 711, 118 (2002). K. Kumerički, D. Müller and K. PassekKumerički, Nucl. Phys. B 794, 244 (2008). K. Kumerički and D. Müller, Nucl. Phys. B 841, 1 (2010). M. Guidal, H. Moutarde and M. Vanderhaeghen, Rept. Prog. Phys. 76, 066202 (2013). D. Müller, M. V. Polyakov and K. M. Semenov-Tian-Shansky, JHEP 1503, 052 (2015). D. Müller and K. M. Semenov-Tian-Shansky, Phys. Rev. D 92, 074025 (2015).

[8] X. D. Ji, W. Melnitchouk and X. Song, Phys. Rev. D 56, 5511 (1997). 
[9] V. Y. Petrov et al., Phys. Rev. D 57, 4325 (1998). P. Schweitzer et al., Phys. Rev. D 66, 114004 (2002). J. Ossmann et al., Phys. Rev. D 71, 034011 (2005). M. Wakamatsu, Phys. Lett. B 648, 181 (2007).

[10] K. Goeke et al., Phys. Rev. D 75, 094021 (2007).

[11] K. Goeke et al., Phys. Rev. C 75, 055207 (2007).

[12] C. Cebulla et al., Nucl. Phys. A 794, 87 (2007). J. H. Jung, U. Yakhshiev and H. C. Kim, J. Phys. G 41, 055107 (2014).

[13] H. C. Kim, P. Schweitzer and U. Yakhshiev, Phys. Lett. B 718, 625 (2012). J. H. Jung, U. Yakhshiev, H. C. Kim and P. Schweitzer, Phys. Rev. D 89, 114021 (2014).

[14] D. Müller and D. S. Hwang, arXiv:1108.3869 [hep-ph].

[15] B. Pasquini, M. V. Polyakov and M. Vanderhaeghen, Phys. Lett. B 739, 133 (2014).

[16] J. F. Donoghue, H. Leutwyler, Z. Phys. C 52, 343 (1991). B. Kubis and U. G. Meissner, Nucl. Phys. A 671, 332 (2000) [Erratum-ibid. A 692, 647 (2001)]. J. W. Chen and X. D. Ji, Phys. Rev. Lett. 88, 052003 (2002) A. V. Belitsky and X. D. Ji, Phys. Lett. B 538, 289 (2002) S.-I. Ando, J.-W. Chen and C.-W. Kao, Phys. Rev. D 74, 094013 (2006). M. Diehl, A. Manashov and A. Schäfer, Eur. Phys. J. A 29, 315 (2006). N. Kivel, M. V. Polyakov and A. Vladimirov, Phys. Rev. D 79, 014028 (2009) A. M. Moiseeva and A. A. Vladimirov, Eur. Phys. J. A 49, 23 (2013). P. Wein, P. C. Bruns and A. Schäfer, Phys. Rev. D 89, 116002 (2014).

[17] E. Megias, E. Ruiz Arriola, L. L. Salcedo and W. Broniowski, Phys. Rev. D 70, 034031 (2004) E. Megias, E. Ruiz Arriola and L. L. Salcedo, Phys. Rev. D 72, 014001 (2005). W. Broniowski and E. R. Arriola, Phys. Rev. D 78, $094011(2008)$

[18] N. Mathur, S. J. Dong, K. F. Liu, L. Mankiewicz and N. C. Mukhopadhyay, Phys. Rev. D 62, 114504 (2000). P. Hägler et al. [LHPC collaboration], Phys. Rev. D 68, 034505 (2003); Phys. Rev. D 77, 094502 (2008). M. Göckeler et al. [QCDSF Collaboration], Phys. Rev. Lett. 92, 042002 (2004). J. D. Bratt et al., Phys. Rev. D 82, 094502 (2010).

[19] S. Liuti, S. K. Taneja, Phys. Rev. C 72, 032201 (2005). V. Guzey and M. Siddikov, J. Phys. G 32, 251 (2006).

[20] I. R. Gabdrakhmanov and O. V. Teryaev, Phys. Lett. B 716, 417 (2012).

[21] M. Mai, P. Schweitzer, Phys. Rev. D 86, 076001 (2012).

[22] M. Mai, P. Schweitzer, Phys. Rev. D 86, 096002 (2012).

[23] R. Friedberg, T. D. Lee and A. Sirlin, Phys. Rev. D 13, 2739 (1976). 
[24] S. R. Coleman, Nucl. Phys. B 262, 263 (1985) [Erratum-ibid. B 269, 744 (1986)].

[25] A. M. Safian, S. R. Coleman and M. Axenides, Nucl. Phys. B 297, 498 (1988).

[26] A. G. Cohen, S. R. Coleman, H. Georgi and A. Manohar, Nucl. Phys. B 272, 301 (1986).

[27] M. G. Alford, Nucl. Phys. B 298, 323 (1988).

[28] T. D. Lee and Y. Pang, Phys. Rept. 221, 251 (1992).

[29] A. Kusenko, Phys. Lett. B 404, 285 (1997); Phys. Lett. B 405, 108 (1997); Phys. Lett. B 406, 26 (1997). A. Kusenko and M. E. Shaposhnikov, Phys. Lett. B 418, 46 (1998).

[30] S. Kasuya and M. Kawasaki, Phys. Rev. D 61, 041301 (2000). K. Enqvist, S. Kasuya and A. Mazumdar, Phys. Rev. D 66, 043505 (2002).

[31] T. Multamaki and I. Vilja, Nucl. Phys. B 574, 130 (2000). F. Paccetti Correia and M. G. Schmidt, Eur. Phys. J. C 21, 181 (2001). T. A. Ioannidou, A. Kouiroukidis and N. D. Vlachos, J. Math. Phys. 46, 042306 (2005).

[32] S. S. Clark, Nucl. Phys. B 756, 38 (2006). M. Fairbairn, A. C. Kraan, D. A. Milstead, T. Sjostrand, P. Skands and T. Sloan, Phys. Rept. 438, 1 (2007).

[33] D. P. Clougherty, Phys. Rev. Lett. 96, 045703 (2006).

[34] M. Schmid and M. Shaposhnikov, Nucl. Phys. B 775, 365 (2007).

[35] Y. Verbin, Phys. Rev. D 76, 085018 (2007). B. Hartmann and J. Riedel, Phys. Rev. D 86, 104008 (2012).

[36] M. S. Volkov and E. Wohnert, Phys. Rev. D 66, 085003 (2002). B. Kleihaus, J. Kunz and M. List, Phys. Rev. D 72, 064002 (2005).

[37] M. Gleiser and J. Thorarinson, Phys. Rev. D 73, 065008 (2006). M. Gleiser and D. Sowinski, Phys. Lett. B 727, 272 (2013).

[38] V. A. Gani, N. B. Konyukhova, S. V. Kurochkin, and V. A. Lensky, Comput. Math. Math. Phys. 44, 1968 (2007).

[39] N. Sakai and M. Sasaki, Prog. Theor. Phys. 119, 929 (2008). T. Tamaki and N. Sakai, Phys. Rev. D 81, 124041 (2010). N. Sakai, H. Ishihara and K.-I. Nakao, Phys. Rev. D 84, 105022 (2011)

[40] M. I. Tsumagari, E. J. Copeland, and P. M. Saffin, Phys. Rev. D 78, 065021 (2008). E. J. Copeland, M. I. Tsumagari, Phys. Rev. D 80, 025016 (2009).

[41] P. Bowcock, D. Foster and P. Sutcliffe, J. Phys. A 42, 085403 (2009). 
[42] H. Arodz and J. Lis, Phys. Rev. D 77, 107702 (2008); Phys. Rev. D 79, 045002 (2009).

[43] G. Gabadadze and R. A. Rosen, Phys. Lett. B 666, 277 (2008).

[44] L. Campanelli and M. Ruggieri, Phys. Rev. D 80, 036006 (2009).

[45] E. Krylov, A. Levin and V. Rubakov, Phys. Rev. D 87, 083528 (2013).

[46] E. Y. Nugaev, M. N. Smolyakov, JHEP 1407, 009 (2014). I. E. Gulamov, E. Y. Nugaev and M. N. Smolyakov, Phys. Rev. D 87, 085043 (2013).

[47] C. Herdeiro, E. Radu and H. Runarsson, Phys. Lett. B 739, 302 (2014).

[48] E. Nugaev and A. Shkerin, Phys. Lett. B 747, 287 (2015).

[49] S. Khlebnikov and I. Tkachev, Phys. Rev. D 61, 083517 (2000).

[50] M. von Laue, Ann. Phys. (Leipzig) 340, 524 (1911). I. Białynicki-Birula, Phys. Lett. A 182, 346 (1993).

[51] N. Graham, Phys. Lett. B 513, 112 (2001).

[52] E. B. Bogomolny, Sov. J. Nucl. Phys. 24, 449 (1976) [Yad. Fiz. 24, 861 (1976)].

[53] M. K. Prasad and C. M. Sommerfield, Phys. Rev. Lett. 35, 760 (1975).

[54] G. 't Hooft, Nucl. Phys. B 79, 276 (1974).

[55] A. M. Polyakov, JETP Lett. 20, 194 (1974) [Pisma Zh. Eksp. Teor. Fiz. 20, 430 (1974)].

[56] H. Georgi and S. L. Glashow, Phys. Rev. Lett. 28, 1494 (1972).

[57] For a pedagogical exposition see: M. Shifman, "Advanced topics in quantum field theory," Cambridge University Press (2012).

[58] F. J. Dyson, Phys. Rev. 85, 631 (1952).

[59] G. V. Dunne and M. Ünsal, JHEP 1211, 170 (2012); Phys. Rev. D 87, 025015 (2013).

[60] K. Enqvist, A. Mazumdar, Phys. Rept. 380, 99 (2003).

[61] O. Teryaev, Nucl. Phys. Proc. Suppl. 245, 195 (2013).

[62] D. Boer et al., report of the joint BNL/INT/JLab program on the science case for an Electron-Ion Collider, report number: INT-PUB-11-034, BNL96164-2011, JLAB-THY-11-1373 [arXiv:1108.1713 [nucl-th]]. 